\title{
Ontario Library Association (OLA) News
}

\section{Knowledge Ontario Successful in Acquiring Province Wide Licenses for a Core Suite of Digital Resources}

The Ontario Ministry of Culture and the Management Group of Knowledge Ontario have announced that negotiations have been successfully completed to supply all publicly funded libraries in the Province of Ontario with a core suite of digital products. The resources contained in these databases will provide access to information that is needed everyday by individual Ontarians and students of all ages. The resources and materials go well beyond what is currently available on the internet, offering full text of newspapers, magazines and books. Access to these databases will be available from wherever people are in Ontario; at home, work, or school. The variety of information to be found in the databases will satisfy the youngest school child, the researcher in a university lab; in fact any citizen of Ontario.

The databases roll out across the province beginning in January. Users authenticated on a library system through the web will have access to the astonishing range of information to be found in the databases. To preview the databases, which will be available through Knowledge Ontario, go to http://access.gale.com/ontario/. In addition to all the Gale databases listed at this site, Knowledge Ontario has also licensed Canadian Reference Centre from Ebsco.

Training sessions on using the databases will take place in regional centres throughout Ontario over the next three months and will be held at OLA Super Conference 2007. Marketing materials, press releases, inhouse training webcams and other materials to aid individual libraries in launching this new service will be made available so that libraries will be ready before the databases go live on January 1, 2007. 